\title{
Performance Analysis of a Backward Reservation Protocol In Networks with Sparse Wavelength Conversion
}

\author{
Lambros Pezoulas, Mark Joseph Francisco \\ Ioannis Lambadaris, Changcheng Huang \\ Carleton University \\ 1125 Colonel By Drive \\ Ottawa, Ont. K1S 5B6
}

\begin{abstract}
In sparse wavelength conversion networks only a few nodes support wavelength conversion. The optical paths in the network consist of a group of segments where each segment independently must meet the wavelength continuity constraint when setting up lightpaths across them. In this paper, we propose a distributed control algorithm called First-Available that can efficiently be used to assign wavelengths in networks with sparse wavelength conversion. The wavelength reservation protocol described is a backward reservation protocol. In previous research it has been found that backward reservation algorithms do not offer much improvement in the case where optical converters are used. First-Available was compared to other backward reservation algorithms such as First-Fit and Random and was shown to outperform those in the case of sparse wavelength conversion. Also, compared to the case of no conversion in the network the use of the First-Available algorithm in combination with using converters gives a lower average blocking probability. In previous papers, we have outlined a method called OBGP to support lightpath setup and management. We have used OBGP to implement and simulate the First-Available algorithm in OPNET. From our simulation results we also collected nodal statistics, and based on these we studied where should be the optimal placement of the converters using the First-Available algorithm.
\end{abstract}

\section{INTRODUCTION}

In recent years extensive research has taken place in the area of WDM networks. Wavelength-routed networks allow to build high bandwidth networks. The performance and the cost of these networks, depend on many design parameters. A performance indication of an All-Optical Network is the call blocking probability[9][11]. The goal is to achieve a low overall blocking probability at an affordable cost, and at the same time to make sure that other critical performance measures are within reasonable values. We will briefly look at some of the design parameters and then look at these in parallel.

One design parameter is the use of Wavelength Converters in an Optical Network. A light-path setup request can be blocked in the absence of a common wavelength across the entire path. This is also known as the wavelength continuity constraint [6][10]. In the case where the network has wavelength converters different wavelengths can be used on each link along the route. Although optical converters lower the average blocking probability, they have a high cost in doing so. Thus, recent work has focused on networks with sparse wavelength conversion[5][7][8]. Networks with sparse wavelength conversion have a small fraction of routing nodes equipped with full wavelength conversion capability. Given that there is a limited number of converters and that their cost is high, efficient use of these converters is very important. Some papers have focused on the optimal placement of converters in a sparse wavelength conversion network such as in [5].

Another, very important design parameter in an AllOptical Network is the choice of wavelength assignment and reservation algorithm. If there are multiple feasible wavelengths between a source node and a destination node, a wavelength assignment algorithm is required to select a wavelength for a given light-path. This is part of a well known problem which is referred to as the Routing and Wavelength Assignment (RWA) problem. For the purposes of this paper we do not concentrate on the routing part of this problem but only the wavelength assignment part. The routing is considered to be fixed routing and is given by the OBGP protocol [1]

Various approaches to the wavelength assignment are possible. A centralized or a distributed approach can be taken in implementing a control protocol for wavelength assignment. As it is mentioned in [2] though, in large networks, centralized control is not realistic, thus a distributed approach to this problem makes more sense. Furthermore, given the distributed approach there are two categories of reservation protocols: Forward Reservation protocols and Backward Reservation. In general it has been shown that backward reservation schemes outperform forward reservation schemes for the case in which there is no wavelength conversion. In the case where there are wavelength converters a forward reservation scheme is more appropriate.

Most of the studies of backward reservation protocols have been done in networks with no conversion. In this paper we will introduce a new backward reservation algorithm called First-Available that can be used in a sparse wavelength conversion network. In many cases a backward reservation protocol such as Random or First-Fit does not add performance improvement when converters are used. We will show how First-Available algorithm actually lowers the blocking probability in the case where converters are used. The performance of this algorithm is compared to other more basic algorithms such as First-Fit and Random, in networks with and without wavelength conversion. The comparison is based on simulations run in OPNET. At the same time we will also study the effect of moving the converters in different nodes in the network and will find where should their optimal placement be, given this reservation protocol. In section II we describe the First-Available wavelength assignment algorithm and highlight the differences from First-Fit and Random approach. In section III we discuss the results that were collected based on our simulations in 
OPNET. Finally in the last section we discuss our conclusions based on our current work.

\section{WAVELENGTH RESERVATION ALGORITHM}

The Control Protocol described in this paper is a backward reservation protocol. The forward path is used for probing the available resources (wavelengths) and the actual reservation takes place in the backward path. This, wavelength assignment protocol supports optical networks with or without optical converters. In the case where there are no wavelength converters in the network the destination node acts as the main decision point, selecting the wavelength to be used for the entire path. In the case where there exist some optical converters, the end-to-end path consists of sub path segments. The endpoints of these segments are defined by the converter node locations. The wavelength continuity constraint is only applied to each individual segment independently. Two separate segments can use different wavelengths. Thus, each node with conversion capability makes a decision for one of the path segments connected to it. The destination is still a decision point for selecting the wavelength of the last segment in the path. As we will see further down the case of no converter is really a special case (i.e. single segment case) of the general algorithm that supports wavelength converters.

The performance of this control protocol depends on the algorithm used for making a wavelength selection at each of the decision points along the path. The algorithm studied in this paper is called First-Available and we compare this to other basic algorithms such as Random selection and FirstFit selection.

The following figure displays a scenario where the path consists of five nodes and there is one converter at node $\mathrm{C}$. Thus, the path consists of two segments. The common wavelengths in segment 1 are $\lambda 1, \lambda 2, \lambda 3$ and in segment 2 $\lambda 4, \lambda 5, \lambda 6$. We will use this diagram to describe our wavelength reservation/selection algorithm.

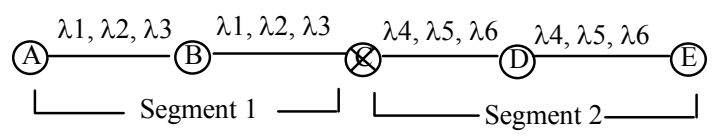

Fig. 1. Two-Segment path

We will first describe how First-Fit backward reservation algorithm works and then describe First-Available and highlight the differences of these two algorithms.

Figure 2 illustrates the First-Fit algorithm. In fig.2a the Discovery message traverses the nodes probing for the available wavelengths. Along with the wavelengths the Discovery message records the segment endpoints on which the wavelengths are available. This is a modification that was made in order to be able to support wavelength converters. Thus, Discovery message 1 finds that $\lambda 1, \lambda 2, \lambda 3$ are available from node $\mathrm{A}$ to node $\mathrm{B}$. When the Discovery message traverses from node $\mathrm{B}$ to $\mathrm{C}$ (Disc.2) the endpoint information in the message is updated as shown in figure $2 \mathrm{a}$ since the Discovery process has discovered that wavelengths $\lambda 1, \lambda 2, \lambda 3$ are available from $\mathrm{A}$ to $\mathrm{C}$.

When a Discovery message reaches a converter node it means that it has reached the end of the current segment and a new segment will be traversed. At this point the converter node needs to select which wavelength to use for the segment that has just been traversed before propagating the Discovery message to the next node. In the First-Fit algorithm the first wavelength from the list of wavelengths for the specific segment is selected. When node $\mathrm{C}$ sends the Discovery message to node $\mathrm{D}$ it only propagates $\lambda 1$ indicating the segment on which it is available (A to $\mathrm{C}$ ) and at the same time it discovers and sends the wavelengths that are available for the new segment $(\lambda 4, \lambda 5, \lambda 6)$. When the Discovery message eventually reaches the destination the destination node needs to select a wavelength to be used for the last segment, similarly to how node $\mathrm{C}$ selected a wavelength for segment $\mathrm{A}-\mathrm{C}$. Thus, $\lambda 4$ is selected at node $\mathrm{E}$ for segment $\mathrm{C}: \mathrm{E}$ and this completes the discovery phase.

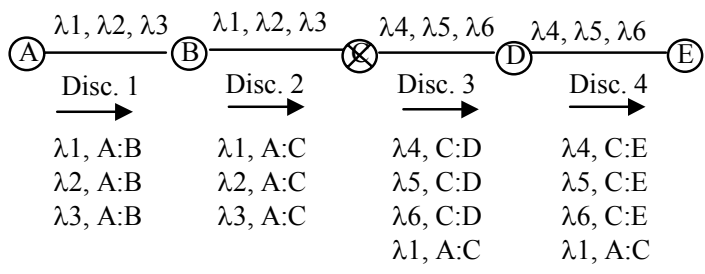

(a)

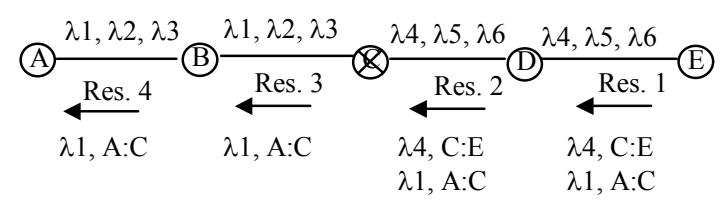

(b)

Fig. 2. First-Fit Wavelength Reservation Algorithm

The Reservation Messages traverse the path in the backward direction as shown in figure $2 \mathrm{~b}$. The wavelengths selected for each segment in the discovery phase are reserved in each node's wavelength table. When the Reservation Message reaches a converter node the wavelength for the previous segment is popped off the list (since the reservation for that segment has completed). For example when $\mathrm{C}$ sends the Reservation Message to $\mathrm{B}$ it only passes $\lambda 1$ and it has completed reservation for segment C-E. Finally, the Reservation Message reaches node $\mathrm{A}$ and a wavelength has successfully been reserved on both segments that consist the path.

The Random selection algorithm is similar to the First-Fit algorithm we just described with the only difference that wavelengths are selected randomly at converter nodes and at the destination node.

Having looked at First-Fit and Random wavelength selection algorithms we will present a new algorithm called First-Available and compare its operation. The message flow for the First-Available algorithm is shown in figure 3 . The first main difference with the other two algorithms is that at a converter node, the wavelengths for the current segment are all passed to the next segment. Thus, a wavelength selection is not made in the forward direction at converter nodes, rather all available wavelengths for each segment are propagated to the destination as shown in figure $3 \mathrm{a}$. As a result though we end up with larger control messages than 
the case of First-Fit and Random. This is easily seen, by comparing the messages passed in figure 2 and figure 3 . The worst case is a network with wavelength converters at every node. In this case the number of light-path segments is equal to the number of links in the path and using the FirstAvailable algorithm would result in significant control overhead. The First-Available algorithm is intended for networks where a small fraction of the nodes have full wavelength conversion capabilities.

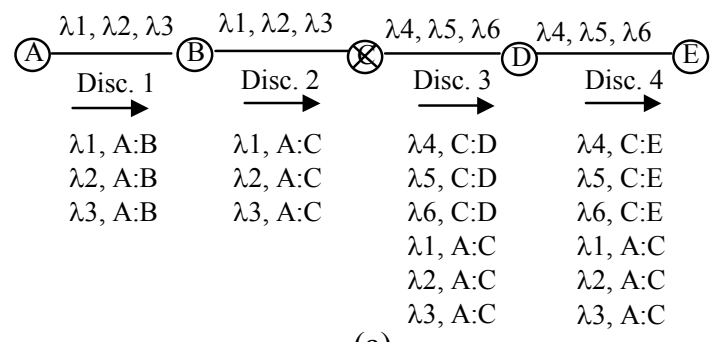

(a)

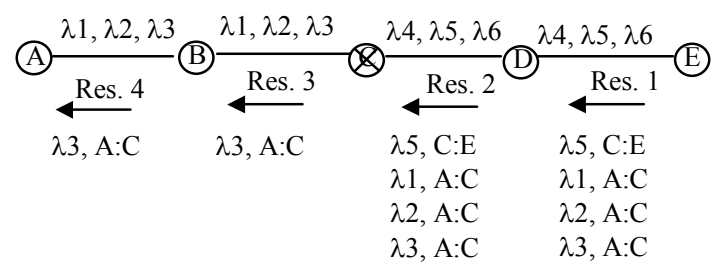

(b)

Fig. 3. First-Available Wavelength Reservation Algorithm

The second difference is in the selection of a wavelength. In the previous algorithms once a wavelength was selected the rest of the wavelengths were discarded from the list. Then in the reservation phase the destination node or a converter node would attempt to reserve the specific wavelength in its wavelength table. In the case where there is competing traffic or multiple requests to the same node it is possible that between the time that discovery phase saw that the selected wavelength was available, and the time it actually tries to reserve the wavelength, that some other request has taken the wavelength and is no longer available. This would result in blocking the initial request.

In order to overcome such blocking First-Available algorithm does not discard the list of wavelengths after selecting a wavelength. It selects the first wavelength from the list and then tries to reserve it at the node where it was selected (destination node or converter node). If the reservation fails, the algorithm attempts to reserve the next wavelength from the list. Retries of wavelength reservations happens only at converter nodes or at the destination. This is repeated until a successful reservation is achieved or if all the wavelengths on the list are attempted and failed. In the later case the request is considered blocked. The First-Available algorithm performs wavelength reservation retries sequentially from the list for simplicity. Alternatively, the wavelength selection retry could be done randomly, but this is not studied in this paper and is slightly more complicated to implement.

In figure $3 b$ we see that the algorithm tried to reserve $\lambda 4$ and failed and then tried $\lambda 5$. Since this was successfully reserved at the destination node this was the wavelength that was propagated to the next node in the path. Similarly, at the converter node $\mathrm{C}$ the algorithm tries to reserve $\lambda 1$ and fails. It then tries to reserve $\lambda 2$ that also fails. Finally it tries $\lambda 3$ and succeeds. This is the wavelength that is then propagated to the rest of the nodes in this segment.

\section{SIMULATION RESULTS}

In order to test the First-Available reservation algorithm and compare with the First-Fit and Random algorithms we had to implement the proper test environment. In this section we see how that was accomplished and then comment on the results that were gathered from our simulations.

\section{A. Implementation}

In order to simulate and compare the performance of the above algorithms the OBGP protocol was used. OBGP which was discussed extensively in [1] is a protocol used to set up dynamic light-paths. The main characteristic of this protocol is that it combines routing and signaling together into one protocol. The AS identifiers of the OBGP routers are used to identify the endpoints of the segments, as well the AS_PATH is also used to determine whether a node is a source node, intermediate or destination node. The AS_PATH also defines the path taken in the backward direction. Using the AS_PATH information, any sourcedestination pair becomes a linear topology. In [1] we also described the wavelength table. Each OBGP router contains a wavelength table containing up-to-date wavelength availability information. This is not end-to-end information but information to the neighbors.

\section{B. Theoretical Analysis}

In order to verify our simulation we perform a simple theoretical analysis of the three algorithms discussed earlier and compare to the simulation results. For our analysis we have considered a linear case with no conversion where we have one traffic stream (i.e. one source node generating requests at a rate of $\lambda \mathrm{req} / \mathrm{sec}$ to a single destination node). The holding time of the light-path is exponentially distributed with average $1 / \mu \mathrm{sec}$. Also in our analysis we take into account link delay since it affects the blocking probability. In [12] an approximate analytical model has been developed which can be used to calculate the blocking probability in more complex networks taking into account propagation delays. The case of wavelength conversion is quite complicated and is not studied in this paper.

1) First-Available Algorithm: Erlang's First Formula can be used to estimate the blocking probability of the FirstAvailable Algorithm. With the First-Available algorithm as long as there is at least one wavelength available there will be no blocking. Also, in contrast with the other algorithms studied First-Available's Blocking Probability in the linear topology does not depend on time delays such as propagation and processing delays. Thus, the following formula allows us to calculate First-Available Blocking in the linear topology:

$$
P_{b l k}=P \text { [lightpath request blocked] }=\frac{\left(\frac{\lambda}{\mu}\right)^{s} / s !}{\sum_{j=0}^{s}\left(\frac{\lambda}{\mu}\right)^{j} / j !}
$$


$s:$ is the number of wavelengths which can be used end-to-end between the source-destination pair. $\lambda$ : the rate at which the source generates lightpath requests to the specific destination.

$\mu$ : the mean holding time of the lightpath after it has been established and setup.

2) First Fit Algorithm: In the case of the First Fit algorithm the blocking probability depends on the propagation delay. This is more evident when requests come very close together.

Let $\quad P_{b l k}=\frac{\left(\frac{\lambda}{\mu}\right)^{s} / s !}{\sum_{j=0}^{s}\left(\frac{\lambda}{\mu}\right)^{j} / j !} \quad$ be the probability that

there is no wavelength available.

Given that the round trip delay between the second last node and the last node in the source-destination path is $d$ time units, then if two lightpath requests come close together by a time difference of less than this propagation delay they will both see that a particular wavelength is available and attempt to reserve it. The first one will successfully reserve it but the second one will fail. Since requests are arriving according to a Poisson distribution with a rate of $\lambda$ req $/ \mathrm{sec}$ the probability that no lightpath requests occur during a time interval of length $\mathrm{d}$ time units is given by:

\section{$P[$ no lightpath requests during time interval $d]=e^{-\lambda d}$}

Thus, the Blocking probability for the First Fit Algorithm can be approximated as follows:

$$
P_{\text {first-fit-blk }}=1-\left(1-P_{b l k}\right) e^{-\lambda d}
$$

3) Random Algorithm: In the case of Random algorithm the blocking probability depends on the propagation delay as was the case with the First Fit algorithm. The effect of time delays is smaller due to the random selection of the wavelength at the destination node.

Let $P b_{[w-k]}$ be the probability that w-k wavelengths are in use. Then we calculate the average number of wavelengths in use as follows:

$$
\text { Avg. number of wavelengths in se }=\sum_{k=0}^{w}(w-k) P b_{[w-k]}
$$

Avg. number of available wavelengths $=$

$$
A w=1-\sum_{k=0}^{w}(w-k) P b_{[w-k]}
$$

Thus, each wavelength gets requests at a rate of $\lambda^{\prime}=$ $\lambda / A w$. Since requests are arriving according to a Poisson distribution with a rate of $\lambda^{\prime} \mathrm{req} / \mathrm{sec}$ the probability that no lightpath requests occur during a time interval of length $d$ time units is given by:

\section{$P[$ no lightpath requests during time interval $d]=e^{-\lambda^{\prime} d}$}

Thus, the Blocking probability for the Random Algorithm can be approximated as follows:

$$
P_{\text {random }}=1-\left(1-P_{b l k}\right) e^{-\lambda^{\prime} d}
$$

Figure 4 shows the theoretical (dotted lines) and simulated (solid lines) blocking probability vs. lightpath request arrival rate graphs for all three algorithms. The figure also shows the $95 \%$ confidence intervals for the simulation graphs.

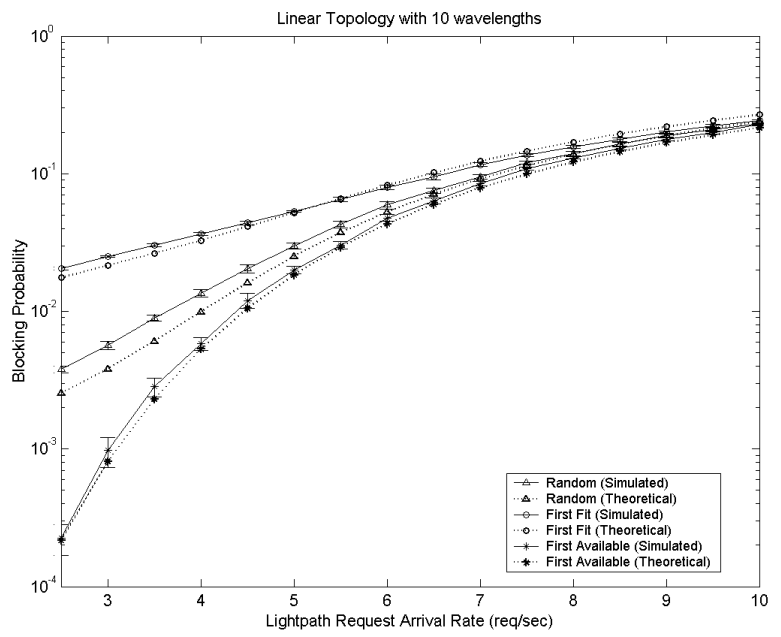

Fig. 4. Blocking Probability graph for Linear Topology with single traffic stream

\section{Simulation Results and Discussion}

In this section we discuss the results obtained from our simulations in OPNET. We observe the performance of the Random, First-Fit, and First-Available algorithms in networks with and without wavelength converters. We also make suggestions as to where the converters should be placed in the network based on nodal statistics gathered in the non-converter scenario. Firstly, we consider the network shown in figure 5. In this scenario node $\mathrm{S}$ makes light-path requests to node $\mathrm{D}$, node $\mathrm{U}$ makes light-path requests to node $\mathrm{W}$, and node $\mathrm{V}$ makes light-path requests to node $\mathrm{Q}$. We have simulated this network with 10 wavelengths on each link. Light-path requests are generated according to a Poisson distribution with a rate of $\lambda \mathrm{req} / \mathrm{sec}$. The holding time of the light-paths is exponentially distributed with a mean of $1 / \mu$ sec.

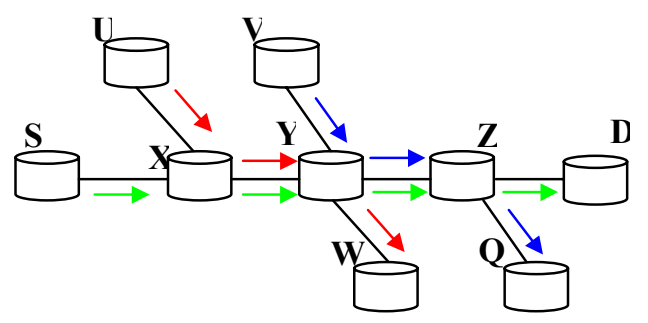

Fig. 5. Network with multiple competing traffic streams.

Initially we looked at the case where there are no wavelength converters in the network. It was observed that at lower light-path request rates, most of the blocking happens due to Reservation Messages being blocked. As the light- 
path setup request arrival rate increases Discovery message blockage starts to contribute to the overall blocking due to lack of resources. The bar graphs in Figure 6 show the percentage of discovery (white bars) and reservation (black bars) messages blocked as the light-path request arrival rate increases, for all three algorithms. At low light-path request arrival rates we see that with the First-Available algorithm (bottom graph) the percentage of Reservation Messages blocked is much higher than the Random (upper left graph) and First Fit algorithm (upper right graph). This happens since the First-Available algorithm as was described earlier reduces the probability of requests being blocked at the destination due to multiple simultaneous requests. As a result more requests make it into the reservation phase where they can get blocked due to competition with other streams.

In our simulations we also measured the number of Discovery and Reservation Messages blocked at each node individually, in order to identify the bottleneck nodes. It was found that all of the Reservation Messages were being blocked at nodes $\mathrm{Y}$ and $\mathrm{Z}$, and the majority of the Discovery messages were being blocked at nodes $\mathrm{X}$ and $\mathrm{Y}$.

In general we found that Reservation Messages happened mostly at nodes that had multiple traffic streams branching out on to different links. This is a result of the fact that reservation happens in the backward path. For example for requests being made to node $\mathrm{Q}$ and node $\mathrm{D}$, as the Discovery messages probe the nodes in the forward direction it is possible that they see the same wavelengths available up to node $\mathrm{Z}$. At this point though the two Discovery messages go onto different links and thus still see the wavelength available. Then at each destination a wavelength is selected and reserved in the backward path. If the two destination nodes choose the same wavelength it is not until the Reservation Message reaches node $\mathrm{Z}$ that it detects the competition and blocks the request. As for the Discovery messages in general wherever there were multiple traffic streams branching in onto a single link, these were the nodes where most of the Discovery messages were blocked.

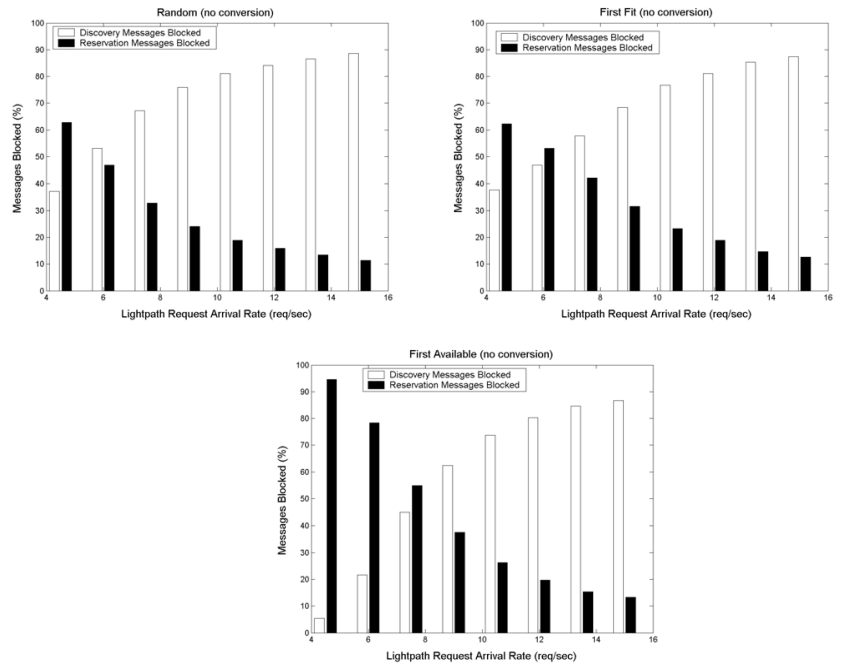

Fig. 6. Message Blockage Comparison (no conversion)

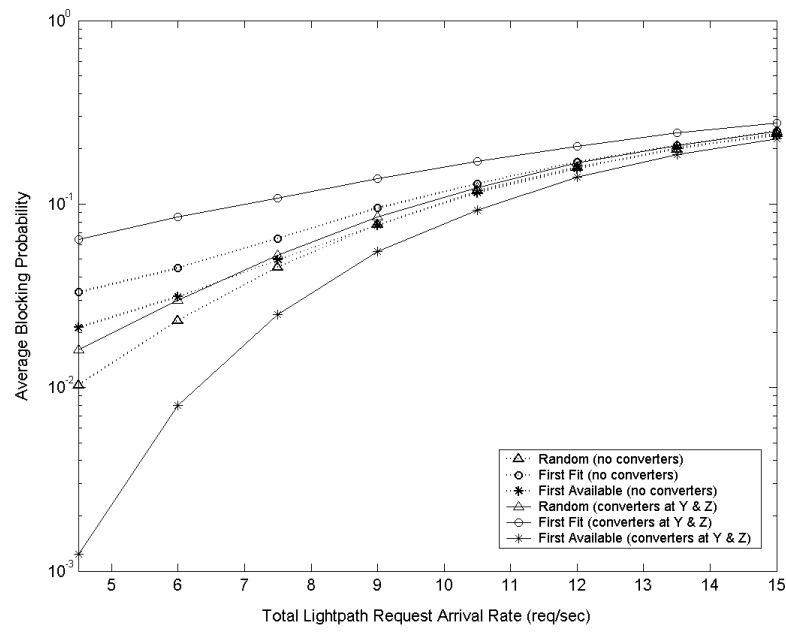

Fig. 7. Comparison of three Algorithms for multiple competing traffic streams.

It was found that a Random selection algorithm performs better than both the First-Fit and First-Available algorithms since there is a smaller probability that the same wavelength is selected at the two destination nodes. The blocking probability for the three algorithms is shown in figure 7 . The dotted lines are the plots of the blocking probability in the case with no wavelength converters in the network. The solid line plots are the plots for the three algorithms in a network with converters at nodes $\mathrm{Y}$ and $\mathrm{Z}$.

After having gathered these results we attempted to make a decision as to where to place wavelength converters in order to make more efficient use of these. From this task it was found that optimal placement of the converters were the nodes where most Reservation Messages were being blocked (nodes $\mathrm{Y}$ and $\mathrm{Z}$ fig. 5). Placing a converter at a node where a large amount of Discovery messages were blocked had no improvement on the overall blocking probability.

After adding the converters at nodes $\mathrm{Y}$ and $\mathrm{Z}$ we compared the performance of the three algorithms. It was found that in the case of a network using wavelength converters the First-Available lowered the blocking probability, while the Random and First-Fit offered no improvement compared to their performance in a network without converters. Furthermore, the First-Available algorithm with converters at nodes $\mathrm{Y}$ and $\mathrm{Z}$, performed much better than the Random algorithm without wavelength converters. These comparisons are also demonstrated in figure 7. Figure 8 also shows the percentage of Discovery and Reservation Messages blocked. From these bar graphs we see that the percentage of Reservation Messages blocked in the First-Available algorithm is very small compared to the Discovery messages blocked. Also, comparing to the case without wavelength converters we see that for low light-path request arrival rates the reservation blockage has greatly been reduced and any blockage is mainly due to Discovery messages blocked. The same though does not apply for the Random and First-Fit algorithms. For Random and First-Fit we see that the percentage of Reservation Messages blocked increases with the use of wavelength converters.

This happens because a converter node as discussed earlier is a decision point where a wavelength is selected for the specific link segment connected to the node. The 
selection is done in the discovery phase, where one wavelength is selected and the rest are discarded in order to reduce control overhead. By the time the Reservation Message reaches the node it is possible that another request has taken the selected wavelength and since only one wavelength was selected while the others were discarded the request gets blocked. In the case of First-Available though it can attempt to reserve any of the wavelengths that were seen available in the forward direction, if the first choice fails, thus reducing blocking probability at converter nodes.

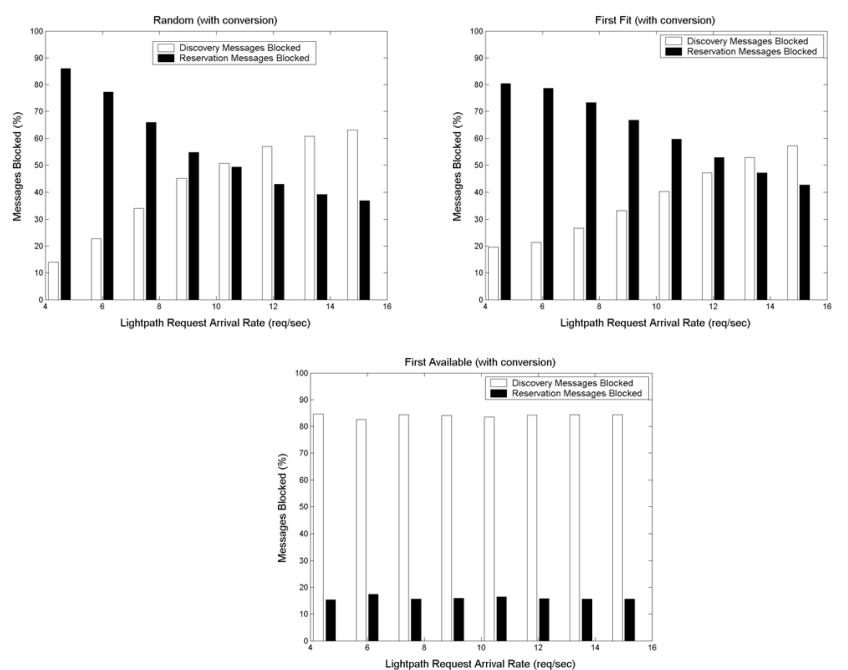

Fig. 8. Message Blockage Comparison with conversion

\section{CONCLUSIONS}

Backward reservation algorithms have been studied by many researchers and have been shown to perform well in networks without wavelength converters. In the case though of networks which have wavelength converters, the use of backward reservation algorithms such as Random and FirstFit do not offer any additional performance improvement as shown in this paper. In this paper we have introduced a new backward reservation algorithm called First-Available which makes better use of the wavelength converters capabilities by lowering the average blocking probability. This is accomplished by allowing the nodes that are considered to be the main decision points for selecting wavelengths to try another wavelength if the first choice does not succeed. Furthermore, First-Available addresses the problem encountered in backward reservation algorithms when multiple connections are set up simultaneously. In this case it is possible that a wavelength available on a link in the forward direction will be taken by a request that comes earlier by a very small time difference. By allowing multiple attempts on different wavelengths, a lower blocking probability is achieved. Although the First-Available algorithm has been implemented into the OBGP Protocol, it can be applied as a general wavelength selection algorithm in other signaling protocols where a limited number of wavelength converters are present.

It was shown in section 2 that the First-Available algorithm introduces extra control overhead compared the other algorithm. This overhead increases with the number of converters thus the networks studied in this paper were networks with sparse wavelength conversion. In such networks the placement of the few converters affects the overall performance of the network. The right choice of where to place the converters is critical. By gathering nodal statistics from the network we found that the nodes that have the highest reservation blockage in the network are the candidate locations for the converters. We further concluded that the nodes with highest reservation blockage are the ones which multiple traffic streams branching out on to multiple links.

\section{REFERENCES}

[1] M. Francisco et al, "Interdomain Routing in Optical Networks", Proceedings of SPIE Opticomm 2001, Vol. 4599, pp 120-129, Denver, August 2001.

[2] X. Yuan, R. Melhem, R. Gupta, Y. Mei, and C. Qiao, "Distributed Control Protocols for Wavelength Reservation and Their Performance Evaluation", Photonic Network Communications, vol. 1, No. 3, 1999 pp. 207-218.

[3] J. Yates, M. Rumswewicz, J.P.R. Lacey, "Wavelength Converters in Dynamically-Reconfigurable WDM Networks", IEEE Communication Surveys, 1999, http://www.comsoc.org/livepubs/surveys/public/2q99i ssue/yates.html.

[4] H. Zang, et al., "A Review of Routing and Wavelength Assignment Approaches for Wavelength-Routed Optical WDM Networks", SPIE Optical Networks Magazine, January 2000.

[5] A. S. Arora, S. Subramaniam, "Wavelength Conversion Placement in WDM Mesh Optical Networks", Photonic Network Communications. Vol. 4, Issue 2, 2002.

[6] D. Banarjee, B. Mukherjee, "A Practical Approach for Routing and Wavelength Assignment in Large Wavelength-Routed Optical Networks", IEEE Journal of Selected Areas in Communications, Vol. 14, pp. 903-908, June 1996.

[7] B. Ramamurthy, B. Mukherjee, "Wavelength Conversion in WDM Networking," IEEE Journal of Selected Areas in Communication, volume 16, pages 1061--1073, September 1996.

[8] S. Subramaniam, M. Azizoglu, A.K. Somani, "Connectivity and Sparse Wavelength Conversion in Wavelength Routing Networks", Proceedings of IEEE Infocom 1996, pp. 148-155, San Francisco 1996.

[9] A. Birman, "Computing approximate blocking probabilities for a class of all-optical networks," IEEE Journal on Selected Areas in Communications, vol. 14, pp. 852-857, June 1996.

[10] J. Fang, R. Srinivasan, and A.K. Somani, "Performance analysis of WDM optical networks with wavelength usage constraint" in the Sixth Working Conference on Optical Networks Design and Modelling, February 2002.

[11] T. Tripathi and K. Sivarajan, "Computing approximate blocking probabilities in wavelength-routed all-optical networks with limited-range wavelength conversion", Proceedings of IEEE Infocom '99, Vol. 1, pp. 329-336, New York, March 1999.

[12] S. Arakawa, K. Miyamoto, M. Murata, and H. Miyahara, "Performance analyses of wavelength reservation methods for high-speed data transfer in photonic networks", Proceedings of ITC-CSCC '99, (Sado), pp.828-831, July 1999. 